\title{
Existence of a Period-Two Solution in Linearizable Difference Equations
}

\author{
E. J. Janowski and M. R. S. Kulenović \\ Department of Mathematics, University of Rhode Island, Kingston, RI 02881-0816, USA \\ Correspondence should be addressed to M. R. S. Kulenović; kulenm@math.uri.edu
}

Received 28 August 2013; Accepted 8 October 2013

Academic Editor: Senada Kalabusic

Copyright (C) 2013 E. J. Janowski and M. R. S. Kulenović. This is an open access article distributed under the Creative Commons Attribution License, which permits unrestricted use, distribution, and reproduction in any medium, provided the original work is properly cited.

\begin{abstract}
Consider the difference equation $x_{n+1}=f\left(x_{n}, \ldots, x_{n-k}\right), n=0,1, \ldots$, where $k \in\{1,2, \ldots\}$ and the initial conditions are real numbers. We investigate the existence and nonexistence of the minimal period-two solution of this equation when it can be rewritten as the nonautonomous linear equation $x_{n+l}=\sum_{i=1-l}^{k} g_{i} x_{n-i}, n=0,1, \ldots$, where $l, k \in\{1,2, \ldots\}$ and the functions $g_{i}: \mathbb{R}^{k+l} \rightarrow \mathbb{R}$. We give some necessary and sufficient conditions for the equation to have a minimal period-two solution when $l=1$.
\end{abstract}

\section{Introduction}

Consider the difference equation

$$
x_{n+1}=f\left(x_{n}, \ldots, x_{n-k}\right), \quad n=0,1, \ldots,
$$

where $k \in\{1,2, \ldots\}$ and the initial conditions are real numbers. We investigate the existence and nonexistence of the minimal period-two solution of this equation when it has the linearization of the form

$$
x_{n+l}=\sum_{i=1-l}^{k} g_{i} x_{n-i}, \quad n=0,1, \ldots,
$$

where $k, l \in\{1,2, \ldots\}$ and the functions $g_{i}: \mathbb{R}^{k+l} \rightarrow \mathbb{R}$. By "(1) has the linearization (2)" we mean that (1) can be rewritten as the nonautonomous linear equation $(2)$; see $[1,2]$.

The importance of a period-two solution is well known in the case of first order difference equations of the form of (1) with $k=0$, where the periods of the solutions appear in the well-known Sharkovsky ordering starting with period two. As a consequence of the results on Sharkovsky ordering the nonexistence of the period-two solution implies the nonexistence of periodic solutions of any period; see [3-5].

In the case of second order difference equations the following result has been obtained in [6].
Theorem 1. Let $I \subseteq R$ and let $f \in C[I \times I, I]$ be a function which either increases in both variables or decreases in the first variable and increases in the second variable. Then for every solution of (1) with $k=1$ the subsequences $\left\{x_{2 n}\right\}_{n=0}^{\infty}$ and $\left\{x_{2 n+1}\right\}_{n=-1}^{\infty}$ of even and odd terms of the solution do exactly one of the following.

(i) Eventually they are both monotonically increasing.

(ii) Eventually they are both monotonically decreasing.

(iii) One of them is monotonically increasing and the other is monotonically decreasing.

As a consequence of Theorem 1 every bounded solution of (1) with $k=1$ approaches either an equilibrium solution, a period-two solution, or a finite point at the boundary, and every unbounded solution is asymptotic to the point at infinity in a monotonic way. In view of Theorem 1 the results on the nonexistence of period-two solutions are as important as the results on the existence of these solutions. The importance of the existence or nonexistence of period-two solutions is also clear from the fact that one of the two most common local bifurcations for second order monotone autonomous difference equations is period-doubling bifurcation; see $[3,4,7$ 9] for related results. Also the only known global bifurcation for second order monotone autonomous difference equations 
is period-doubling bifurcation [10]. See [11-13] for related results. The nonexistence results for periodic solutions which are the discrete analogue of the Bendixson's nonexistence result for periodic solutions of differential equations have been obtained in $[14,15]$.

The results obtained in this paper are applicable to both autonomous and nonautonomous difference equations as the coefficients $g_{i}$ in (2) are in general functions of $n$ and $x_{n-i}, i=$ $0,1, \ldots$. Some of our examples will reflect this situation. The method of finding period-two solutions in the autonomous case consists of finding the fixed points of the second iterate of the corresponding map. However, in the nonautonomous case this method does not work and the results which will be presented in this paper can be used to find period-two solutions.

Some interesting points of our results can be demonstrated by the following example.

Example 2. The period-two solution $\{\Phi, \Psi\}, \Phi \neq \Psi$ of the difference equation

$$
x_{n+1}=a_{n} x_{n}+b_{n} x_{n-1}, \quad n=0,1, \ldots,
$$

where $\left\{a_{n}\right\}$ and $\left\{b_{n}\right\}$ are two real sequences, satisfies

$$
\Phi=a_{n} \Psi+b_{n} \Phi, \quad \Psi=a_{n} \Phi+b_{n} \Psi, \quad n=0,1, \ldots,
$$

which implies

$$
b_{n}-a_{n}=1, \quad n=0,1, \ldots
$$

Conversely, if condition (5) holds, then any possible periodtwo solution $\{\Phi, \Psi\}, \Phi \neq \Psi$ of (3) must satisfy

$$
a_{n}(\Phi+\Psi)=0, \quad n=0,1, \ldots
$$

If $a_{N} \neq 0$ for some $N=0,1, \ldots$ then $\Psi=-\Phi$ and (3) has an infinite number of period-two solutions of the form $\{\Phi,-\Phi\}$, $\Phi \neq 0$. If $a_{n}=0$ for every $n=0,1, \ldots$ then $b_{n}=1$ for every $n=0,1, \ldots$ in which case every nonequilibrium solution of (3) is a period-two solution.

Thus, condition (5) is a necessary and sufficient condition for the existence of a period-two solution. This condition is clearly satisfied if, for instance,

$$
a_{n}=\frac{1}{n+1}-1, \quad b_{n}=\frac{1}{n+1}, \quad n=0,1, \ldots,
$$

or if $\left\{a_{n}\right\}$ and $\left\{b_{n}\right\}$ are period-two sequences which satisfy

$$
\begin{gathered}
b_{2 n}=1, \quad b_{2 n+1}=b, \\
a_{2 n}=0, \quad a_{2 n+1}=b-1, \quad n=0,1, \ldots,
\end{gathered}
$$

or if $\left\{a_{n}\right\}$ and $\left\{b_{n}\right\}$ are period- $k$ sequences which satisfy

$$
b_{n+i}-a_{n+i}=1, \quad i=0,1, \ldots, k-1, \quad n=0,1, \ldots
$$

An example of a nonautonomous nonlinear difference equation for which one can find a period-two solution is the following equation

$$
\begin{aligned}
x_{n+1}= & \left(c_{n}+x_{n-2}+x_{n-4}-1\right) x_{n} \\
& +\left(c_{n}+x_{n-2}+x_{n-4}\right) x_{n-1}, \quad n=0,1, \ldots .
\end{aligned}
$$

The quadratic second order difference equation

$$
\begin{array}{r}
x_{n+1}=A x_{n}^{2}+C x_{n-1}^{2}+D x_{n}+E x_{n-1}, \\
n=0,1, \ldots,
\end{array}
$$

where $A, C, D$, and $E$ are constants, can be linearized as

$$
\begin{array}{r}
x_{n+1}=\left(A x_{n}+D\right) x_{n}+\left(C x_{n-1}+E\right) x_{n-1}, \\
n=0,1, \ldots,
\end{array}
$$

which is of the form of (3) where $a_{n}=A x_{n}+D, b_{n}=C x_{n-1}+$ $E$ and condition (5) becomes the first order linear difference equation

$$
C x_{n-1}+E-A x_{n}-D=1, \quad n=0,1, \ldots
$$

Since $a_{n} \neq 0, n \geq 0$ then the period-two solution of (11) has the form $\left\{x_{-1},-x_{-1}\right\}, x_{-1} \neq 0$.

\section{The Constant Case}

In this section we consider the case when the sums of the even indexed functions $g_{i}$ and the odd indexed functions $g_{i}$ are both constants.

The following simple result will be a useful technical tool.

Lemma 3. Suppose that (1) has the linearization (2). Let

$$
\begin{gathered}
\alpha=\sum_{i \in\{1-l, \ldots, k: i \text { even }\}} g_{i}, \\
\beta=\sum_{i \in\{1-l, \ldots, k: \text { i odd }\}} g_{i}, \quad n=0,1, \ldots
\end{gathered}
$$

Assume that (1) has a minimal period-two solution ..., $\Phi$, $\Psi, \ldots$, where $\Phi \neq \Psi, \Psi=x_{2 n}$, and $\Phi=x_{2 n-1}$ for $n \geq 0$.

(a) For $l \in\{1,3, \ldots\}$,

$$
\begin{aligned}
& \text { if } n \text { is even, then } \Phi(1-\beta)=\Psi \alpha \text {; } \\
& \text { if } n \text { is odd, then } \Psi(1-\beta)=\Phi \alpha .
\end{aligned}
$$

(b) For $l \in\{2,4, \ldots\}$,

$$
\begin{aligned}
& \text { if } n \text { is even, then } \Psi(1-\alpha)=\Phi \beta \\
& \text { if } n \text { is odd, then } \Phi(1-\alpha)=\Psi \beta
\end{aligned}
$$

Proof. By plugging $\Psi=x_{2 n}$ and $\Phi=x_{2 n-1}$ for $n \geq 0$ in (2) and assuming that $l$ is odd we obtain immediately that when $n$ is even (15) holds, while in the case when $n$ is odd (16) holds. Similarly assuming that $l$ is even we obtain immediately that in the case when $n$ is even (17) holds, while when $n$ is odd (18) holds.

Theorem 4. Suppose that (1) has the linearization (2) with $l=1$ and that $\alpha, \beta$ are given by (14). Then (1) has a minimal 
period-two solution $\ldots, \Phi, \Psi, \ldots$, where $\Phi \neq \Psi, \Psi=x_{2 n}$, and $\Phi=x_{2 n-1}$ for $n \geq 0$ if and only if the following hold:

(a) if $n$ is even, then $\Phi(1-\beta)=\Psi \alpha$;

(b) if $n$ is odd, then $\Psi(1-\beta)=\Phi \alpha$.

Proof. The necessary part of the proof follows from part (a) of Lemma 3.

For the proof of the sufficient part choose the initial conditions $\Psi=x_{0}=x_{-2}=\cdots, \Phi=x_{-1}=x_{-3}=\cdots$ and $x_{0} \neq x_{-1}$. Setting $n=0,1, \ldots$ we get that, by part (a),

$$
\begin{aligned}
& x_{1}=\alpha \Psi+\beta \Phi=\Phi, \\
& x_{2}=\alpha \Phi+\beta \Psi=\Psi, \\
& x_{3}=\alpha \Psi+\beta \Phi=\Phi, \\
& x_{4}=\alpha \Phi+\beta \Psi=\Psi .
\end{aligned}
$$

By using induction we get that $\Phi \neq \Psi, \Psi=x_{2 n}$, and $\Phi=$ $x_{2 n-1}$ for $n \geq 0$.

Theorem 5. Suppose that (1) has the linearization (2) and that $\alpha, \beta$ are given by (14). Assume that (1) has a minimal periodtwo solution ..., $\Phi, \Psi, \ldots$, where $\Phi \neq \Psi, \Psi=x_{2 n}$, and $\Phi=$ $x_{2 n-1}$ for $n \geq 0$.

(1) If $l \in\{1,3, \ldots\}$, then

(a) $\beta=1$ if and only if $\alpha=0$;

(b) if $\beta \neq 1$, then $\beta-\alpha=1$ and $\Psi=-\Phi$.

(2) If $l \in\{2,4, \ldots\}$, then

(a) $\alpha=1$ if and only if $\beta=0$;

(b) if $\alpha \neq 1$, then $\alpha-\beta=1$ and $\Psi=-\Phi$.

Proof. The proof is as follows.

(1) Let $l \in\{1,3, \ldots\}$. In view of Lemma 3 part (a) the identities (15) and (16) are satisfied.

(a) Assume that $\beta=1$. Then by (15) $\Psi \alpha=0$. Hence either $\Psi=0$ or $\alpha=0$. If $\Psi=0$, then by (16) $\Phi \alpha=0$, and since $\Phi \neq 0$, we have $\alpha=0$.

Assume that $\alpha=0$. Then by (15) $\Phi(1-\beta)=0$. Hence either $\Phi=0$ or $\beta=1$. If $\Phi=0$, then by $(16) \Psi(1-\beta)=0$, and since $\Psi \neq 0$, we obtain that $\beta=1$.

(b) Assume that $\beta \neq 1$. Then by (15) and (16) $\Phi^{2}(1-$ $\beta)=\Phi \Psi \alpha=\Psi^{2}(1-\beta)$. Thus $\Phi^{2}=\Psi^{2}$ and so $\Psi=-\Phi$. By $(15) \Phi(1-\beta)=-\Phi \alpha$, and since $\Phi \neq 0$, then $\beta-\alpha=1$.

(2) Let $l \in\{2,4, \ldots\}$. In view of Lemma 3 part (b) the identities (17) and (18) are satisfied.

(a) Assume that $\alpha=1$. Then by (17) $\Phi \beta=0$. Hence either $\Phi=0$ or $\beta=0$. If $\Phi=0$, then by (18) $\Psi \beta=0$, and since $\Psi \neq 0$, we have $\beta=0$.
Conversely, assume that $\beta=0$. Then by (17) $\Psi(1-\alpha)=0$. Hence either $\Psi=0$ or $\alpha=1$. If $\Psi=0$, then by (18) $\Phi(1-\alpha)=0$, and since $\Phi \neq 0$, we obtain that $\alpha=1$.

(b) Assume that $\alpha \neq 1$. Then by (17) and (18) $\Phi^{2}(1-$ $\alpha)=\Phi \Psi \beta=\Psi^{2}(1-\alpha)$. Thus $\Phi^{2}=\Psi^{2}$ and so $\Psi=-\Phi$. By $(17)-\Phi(1-\alpha)=\Phi \beta$, and since $\Phi \neq 0$, then $\alpha-\beta=1$.

Theorem 6. Suppose that (1) has the linearization (2) with $l=$ 1 and that $\alpha, \beta$ are given by (14). Then (1) has a minimal periodtwo solution if and only if

$$
\beta-\alpha=1
$$

Proof. If (1) has a minimal period-two solution then by Theorem 5 part (1) the necessary condition follows.

Conversely, assume that $\beta=1$. Then $\alpha=0$. Choose the initial conditions

$$
x_{0}=x_{-2}=\cdots, \quad x_{-1}=x_{-3}=\cdots, \quad x_{0} \neq x_{-1} .
$$

Then for $n=0$ we get $x_{1}=\alpha x_{0}+\beta x_{-1}=x_{-1}$ and for $n=1$ we get $x_{2}=\alpha x_{-1}+\beta x_{0}=x_{0}$, which shows that $\left\{x_{n}\right\}$ is a minimal period-two solution.

Now suppose that $\beta \neq 1$. Choose the initial conditions

$$
x_{0}=x_{-2}=\cdots, \quad x_{-1}=x_{-3}=\cdots, \quad x_{0}=-x_{-1} .
$$

Then for $n=0$ we get $x_{1}=\alpha x_{0}+\beta x_{-1}=(\beta-\alpha) x_{-1}=x_{-1}$ and for $n=1$ we get $x_{2}=\alpha x_{-1}+\beta x_{0}=(\beta-\alpha) x_{0}=x_{0}$, which shows that $\left\{x_{n}\right\}$ is a minimal period-two solution.

An immediate consequence of Theorem 6 is this result.

Corollary 7. (a) If $\beta=1$ and $\alpha=0$, then the minimal periodtwo solution of (1) is ..., $\Psi, \Phi, \ldots$.

(b) If $\beta \neq 1$ and $\beta-\alpha=1$, then the minimal period-two solution of $(1)$ is either ..., $-\Phi, \Phi, \ldots$ or . . ., $-\Psi, \Psi, \ldots$.

\section{The Nonconstant Case}

In this section we consider the case when the sums of the even indexed functions $g_{i}$ and the odd indexed functions $g_{i}$ are both nonconstants.

Theorem 8. Suppose that (1) has the linearization (2) with $l=$ 1. Let

$$
\begin{gathered}
A_{n}=\sum_{i \in\{1-l, \ldots, k ; \text { i iven }\}} g_{i}, \\
B_{n}=\sum_{i \in\{1-l, \ldots, k ; \text { iod } d\}} g_{i}, \quad n=0,1, \ldots .
\end{gathered}
$$

Then (1) has a minimal period-two solution ..., $\Phi, \Psi, \ldots$, where $\Phi \neq \Psi, \Psi=x_{2 n}$, and $\Phi=x_{2 n-1}$ for $n \geq 0$ if and only if $B_{n}-A_{n}=1, n=0,1, \ldots$

Proof. The proof follows from the same reasoning as in Example 2. 
Note that it is possible for (3) to have a minimal periodtwo solution other than $\left\{x_{-1},-x_{-1}\right\}, x_{-1} \neq 0$, when $a_{n} \neq 0$ for all $n \geq 0$ (see Example 12). In order to handle the cases not covered by Theorem 8 we establish the following results.

The following simple result will be a useful technical tool.

Lemma 9. Suppose that (1) has the linearization (2) and that $A_{n}, B_{n}$ are given by (23). Assume that (1) has a minimal periodtwo solution ..., $\Phi, \Psi, \ldots$, where $\Phi \neq \Psi, \Psi=x_{2 n}$, and $\Phi=$ $x_{2 n-1}$ for $n \geq 0$. Then for $n=0,1, \ldots$,

(a) if $l \in\{1,3, \ldots\}$, then

$$
\begin{gathered}
\Phi\left(1-B_{2 n}\right)=\Psi A_{2 n}, \\
\Psi\left(1-B_{2 n+1}\right)=\Phi A_{2 n+1} ;
\end{gathered}
$$

(b) if $l \in\{2,4, \ldots\}$, then

$$
\begin{gathered}
\Psi\left(1-A_{2 n}\right)=\Phi B_{2 n}, \\
\Phi\left(1-A_{2 n+1}\right)=\Psi B_{2 n+1} .
\end{gathered}
$$

Proof. Assume that $l$ is odd. By plugging $\Psi=x_{2 n}, \Phi=x_{2 n-1}$ for $n \geq 0$ in (2) and setting $n=0,1, \ldots$ we obtain immediately that

$$
\begin{gathered}
x_{l}=A_{0} \Psi+B_{0} \Phi=\Phi, \\
x_{l+1}=A_{1} \Phi+B_{1} \Psi=\Psi, \\
x_{l+2}=A_{2} \Psi+B_{2} \Phi=\Phi, \\
x_{l+3}=A_{3} \Phi+B_{3} \Psi=\Psi .
\end{gathered}
$$

Now simple induction completes the proof of (24) and (25) and so of part (a). The proof of part (b) is similar.

Theorem 10. Suppose that (1) has the linearization (2) with $l=1$ and that $A_{n}, B_{n}$ are given by (23). Then (1) has a minimal period-two solution..., $\Phi, \Psi, \ldots$, where $\Phi \neq \Psi, \Psi=$ $x_{2 n}$, and $\Phi=x_{2 n-1}$ for $n \geq 0$ if and only if (24) and (25) hold.

Proof. The necessary part of the proof follows from part (a) of Lemma 9.

For the proof of sufficient part choose the initial conditions $\Psi=x_{0}=x_{-2}=\cdots, \Phi=x_{-1}=x_{-3}=\cdots$, and $x_{0} \neq x_{-1}$.

(1) Assume $\Phi, \Psi \neq 0$. Setting $n=0,1, \ldots$ we get that

$$
\begin{aligned}
& \text { by (24) } x_{1}=A_{0} \Psi+B_{0} \Phi=\Phi, \\
& \text { by (25) } x_{2}=A_{1} \Phi+B_{1} \Psi=\Psi, \\
& \text { by (24) } x_{3}=A_{2} \Psi+B_{2} \Phi=\Phi, \\
& \text { by (25) } x_{4}=A_{3} \Phi+B_{3} \Psi=\Psi .
\end{aligned}
$$

By using induction we get that $\Phi \neq \Psi, \Psi=x_{2 n}$, and $\Phi=x_{2 n-1}$ for $n \geq 0$.

(2) Assume $\Psi=0$. We obtain from (24) and (25) that $B_{2 k}=1$ and $A_{2 k+1}=0$ for $k=0,1, \ldots$, which implies that $x_{2 n}=0$ and $x_{2 n+1}=\Phi, n=0,1, \ldots$
(3) Assume $\Phi=0$. We obtain from (24) and (25) that $A_{2 k}=0$ and $B_{2 k+1}=1$ for $k=0,1, \ldots$, which implies that $x_{2 n+1}=0$ and $x_{2 n}=\Psi, n=0,1, \ldots$

When (1) has been embedded into a higher order equation, the following results can be used to establish either the nonexistence or the necessary conditions for existence of a minimal period-two solution.

Theorem 11. Let $l \in\{1,3, \ldots\}$. Suppose that (1) has the linearization (2) and that $A_{n}, B_{n}$ are as in (23). Assume that (1) has a minimal period-two solution ..., $\Phi, \Psi, \ldots$, where $\Phi \neq \Psi, \Psi=x_{2 n}$, and $\Phi=x_{2 n-1}$ for $n \geq 0$.

(1) Let either $B_{n} \neq 1$ or $A_{n} \neq 0$ for $n \geq 0$; then

(a) $\Phi / \Psi=A_{0} /\left(1-B_{0}\right)=A_{2 n} /\left(1-B_{2 n}\right), n=$ $0,1, \ldots$,

(b) $\Psi / \Phi=A_{1} /\left(1-B_{1}\right)=A_{2 n+1} /\left(1-B_{2 n+1}\right), n=$ $0,1, \ldots$

(2) Let $B_{n}=1$ for $n \geq 0$.

(a) If $A_{2 n}=A_{2 n+1}, n=0,1, \ldots$, then $A_{2 n}=$ $A_{2 n+1}=0, n=0,1, \ldots$.

(b) If $A_{2 n} \neq A_{2 n+1}, n=0,1, \ldots$, then the following are true:

(i) $A_{2 n} \neq 0$ if and only if $A_{2 n+1}=0$ for $n=$ $0,1, \ldots$;

(ii) $A_{2 n}=0$ if and only if $A_{2 n+1} \neq 0$ for $n=$ $0,1, \ldots$;

(iii) either $\Psi=0$ or $\Phi=0$.

(3) Let $A_{n}=0$ for $n \geq 0$.

(a) If $B_{2 n}=B_{2 n+1}, n=0,1, \ldots$, then $B_{2 n}=B_{2 n+1}=$ $1, n=0,1, \ldots$

(b) If $B_{2 n} \neq B_{2 n+1}, n=0,1, \ldots$, then the following are true:

(i) $B_{2 n} \neq 1$ if and only if $B_{2 n+1}=1$ for $n=$ $0,1, \ldots$;

(ii) $B_{2 n}=1$ if and only if $B_{2 n+1} \neq 1$ for $n=$ $0,1, \ldots$;

(iii) either $\Psi=0$ or $\Phi=0$.

Proof. The proof is as follows.

(1) Assume that $B_{n} \neq 1$ for $n \geq 0$. Then $\Phi, \Psi \neq 0$. Otherwise, suppose that $\Psi=0$. Then $\Phi \neq 0$ and in view of (24) $\Phi\left(1-B_{2 n}\right)=0$. This implies $B_{2 n}=1$ for $n \geq 0$, which is a contradiction. Now, suppose that $\Phi=0$. Then $\Psi \neq 0$ and in view of $(25) \Psi\left(1-B_{2 n+1}\right)=$ 0 . This implies $B_{2 n+1}=1$ for $n \geq 0$, which is a contradiction.

Thus $\Phi, \Psi \neq 0$, which in view of Lemma 9, implies $A_{n} \neq 0$ for $n \geq 0$.

(a) By (24) $\Phi / \Psi=A_{0} /\left(1-B_{0}\right)=A_{2 n} /(1-$ $\left.B_{2 n}\right), n=0,1, \ldots$ 
(b) By (25) $\Psi / \Phi=A_{1} /\left(1-B_{1}\right)=A_{2 n+1} /(1-$ $\left.B_{2 n+1}\right), n=0,1, \ldots$

Next, assume that $A_{n} \neq 0$ for $n \geq 0$. Then $\Phi, \Psi \neq 0$. Otherwise, suppose that $\Psi=0$. Then $\Phi \neq 0$ and in view of (25) $\Phi A_{2 n+1}=0$. This implies $A_{2 n+1}=0$ for $n \geq 0$, which is a contradiction. Now, suppose that $\Phi=0$. Then $\Psi \neq 0$ and in view of (24) $\Psi A_{2 n}=0$. This implies $A_{2 n}=0$ for $n \geq 0$, which is a contradiction.

Thus $\Phi, \Psi \neq 0$, which in view of Lemma 9 , implies $B_{n} \neq 1$ for $n \geq 0$.

The rest of the proof is similar to the first part of the proof and will be omitted.

(2) In view of (24) and (25) the condition $B_{n}=1$ for $n \geq 0$ implies $A_{2 n} \Psi=A_{2 n+1} \Phi=0$ for $n=0,1, \ldots$

(a) Assume that $A_{2 n}=A_{2 n+1}, n=0,1, \ldots$

If $A_{2 n} \neq 0$ for some $n \geq 0$, then $A_{2 n+1} \neq 0$ for this $n$ and so $\Phi=\Psi=0$, which is a contradiction. Thus $A_{2 n}=0$ for $n \geq 0$, and so $A_{2 n}=A_{2 n+1}=$ $0, n=0,1, \ldots$

(b) Assume that $A_{2 n} \neq A_{2 n+1}$ for $n \geq 0$.

(i) If $A_{2 n+1}=0$ for $n \geq 0$, then $A_{2 n} \neq 0$ for $n \geq 0$. Now suppose that $A_{2 n} \neq 0$ for $n \geq 0$; then from $A_{2 n} \Psi=A_{2 n+1} \Phi=0$ we get that $\Psi=\Phi A_{2 n+1} / A_{2 n}=0$ for $n \geq 0$.

Since $\Psi=0$, then $\Phi \neq 0$. Thus $A_{2 n+1}=0$ for $n \geq 0$.

(ii) The proof is similar to part (i) and will be omitted.

(iii) By (24) $A_{2 n} \Psi=0$ for $n \geq 0$. Now suppose that $A_{2 n} \neq 0$ for $n \geq 0$, then $\Psi=0$.

If $A_{2 n}=0$ for $n \geq 0$; then $A_{2 n+1} \neq 0$ for $n \geq 0$. In view of (25) $A_{2 n+1} \Phi=0$ for $n \geq 0$ and so $\Phi=0$.

(3) In view of (24) and (25) condition $A_{n}=0$ for $n \geq 0$ implies $\left(1-B_{2 n}\right) \Phi=\left(1-B_{2 n+1}\right) \Psi=0$ for $n=0,1, \ldots$.

(a) Assume that $B_{2 n}=B_{2 n+1}, n=0,1, \ldots$.

If $B_{2 n} \neq 1$ for some $n \geq 0$, then $B_{2 n+1} \neq 1$ for this $n$ and so $\Phi=\Psi=0$, which is a contradiction. Thus $B_{2 n}=1$ for $n=0,1, \ldots$ and consequently $B_{2 n}=B_{2 n+1}=1, n=0,1, \ldots$

(b) Assume that $B_{2 n} \neq B_{2 n+1}$ for $n \geq 0$.

(i) If $B_{2 n+1}=1$ for $n \geq 0$, then $B_{2 n} \neq 1$ for $n \geq 0$. Now suppose that $B_{2 n} \neq 1$ for $n \geq 0$; then from $\left(1-B_{2 n}\right) \Phi=\left(1-B_{2 n+1}\right) \Psi=0$ we get that $\Phi=\Psi\left(1-B_{2 n+1}\right) /\left(1-B_{2 n}\right)=0$ for $n \geq 0$. Since $\Phi=0$, then $\Psi \neq 0$. Thus $B_{2 n+1}=1$ for $n \geq 0$.

(ii) The proof is similar to part (i) and will be omitted.

(iii) By (24) $\left(1-B_{2 n}\right) \Phi=0$ for $n \geq 0$. Now suppose that $B_{2 n} \neq 1$ for $n \geq 0$, then $\Phi=0$. If $B_{2 n}=1$ for $n \geq 0$, then $B_{2 n+1} \neq 1$ for $n \geq 0$. In view of (25) $\Psi\left(1-B_{2 n+1}\right)=0$ for $n \geq 0$ and so $\Psi=0$. (i) If $B_{2 n+1}=0$ for $n \geq 0$; then $B_{2 n} \neq 0$ for $n \geq 0$. Now suppose that $B_{2 n} \neq 0$ for $n \geq 0$; then from $B_{2 n} \Phi=B_{2 n+1} \Psi=0$ we get that $\Phi=$ $\Psi B_{2 n+1} / B_{2 n}=0$ for $n \geq 0$. Since $\Phi=0$, then $\Psi \neq 0$. Thus $B_{2 n+1}=0$ for $n \geq 0$.

(ii) The proof is similar to the proof of part (i).

(iii) By (26) $B_{2 n} \Phi=0$ for $n \geq 0$. If $B_{2 n} \neq 0$ for $n \geq 0$, then $\Phi=0$. Now if $B_{2 n}=0$ for $n \geq 0$, then $B_{2 n+1} \neq 0$ for $n \geq 0$. In view of (27) $\Psi B_{2 n+1}=0$ for $n \geq 0$ and so $\Psi=0$.

Example 12. The difference equation

$$
x_{n+1}=\frac{c_{n}}{n+1} x_{n}+\left(1-\frac{1}{n+1}\right) x_{n-1}, \quad n=0,1, \ldots
$$

where $\left\{c_{n}\right\}$ is a period-two sequence such that $c_{2 n}=\gamma \neq 0,1$, $c_{2 n+1}=1 / \gamma$, has an infinite number of period-two solutions of the form $\left\{x_{-1}, x_{-1} / \gamma\right\}, x_{-1} \neq 0$, which can be seen by immediate checking.

This equation is an illustration of Theorem 11 part 1 . In this case $A_{n}=\{\gamma, 1 / 2 \gamma, \gamma / 3,1 / 4 \gamma, \gamma / 5, \ldots\}$ and $B_{n}=$ $\{0,1 / 2,2 / 3,3 / 4,4 / 5, \ldots\}$.

Theorem 13. Let $l \in\{2,4, \ldots\}$. Suppose that (1) has the linearization (2) and $A_{n}, B_{n}$ are as in (23). Assume that (1) has a minimal period-two solution ..., $\Phi, \Psi, \ldots$, where $\Phi \neq \Psi$, $\Psi=x_{2 n}$, and $\Phi=x_{2 n-1}$ for $n \geq 0$.

(1) Let either $A_{n} \neq 1$ or $B_{n} \neq 0$ for $n \geq 0$; then

(a) $\Psi / \Phi=B_{0} /\left(1-A_{0}\right)=B_{2 n} /\left(1-A_{2 n}\right), n=$ $0,1, \ldots$,

(b) $\Phi / \Psi=B_{1} /\left(1-A_{1}\right)=B_{2 n+1} /\left(1-A_{2 n+1}\right), n=$ $0,1, \ldots$.

(2) Let $A_{n}=1$ for $n \geq 0$.

(a) If $B_{2 n}=B_{2 n+1}, n=0,1, \ldots$, then $B_{2 n}=B_{2 n+1}=$ $0, n \geq 0$.

(b) If $B_{2 n} \neq B_{2 n+1}, n=0,1, \ldots$, then the following are true:

(i) $B_{2 n} \neq 0$ if and only if $B_{2 n+1}=0$ for $n=0$, $1, \ldots$;

(ii) $B_{2 n}=0$ if and only if $B_{2 n+1} \neq 0$ for $n=0$, $1, \ldots$;

(iii) either $\Psi=0$ or $\Phi=0$.

(3) Let $B_{n}=0$ for $n \geq 0$.

(a) If $A_{2 n}=A_{2 n+1}, n=0,1, \ldots$, then $A_{2 n}=A_{2 n+1}$ $=1, n \geq 0$.

(b) If $A_{2 n} \neq A_{2 n+1}, n=0,1, \ldots$, then the following are true:

(i) $A_{2 n} \neq 1$ if and only if $A_{2 n+1}=1$ for $n=0$, $1, \ldots$;

(ii) $A_{2 n}=1$ if and only if $A_{2 n+1} \neq 1$ for $n=0$, $1, \ldots$;

(iii) either $\Psi=0$ or $\Phi=0$. 
Proof. The proof is as follows.

(1) Assume that $A_{n} \neq 1$ for $n \geq 0$. Then $\Phi \Psi \neq 0$. Otherwise, if $\Phi=0$ then $\Psi \neq 0$. By (26) $\Psi\left(1-A_{2 n}\right)=0$ and so $A_{2 n}=1$ for $n \geq 0$, which is a contradiction. In the other case, if $\Psi=0$ then $\Phi \neq 0$. By (27) $\Phi(1-$ $\left.A_{2 n+1}\right)=0$ and so $A_{2 n+1}=1$ for $n \geq 0$, which is a contradiction. Hence $B_{n} \neq 0$ for $n \geq 0$.

(a) By (26) $\Psi / \Phi=B_{0} /\left(1-A_{0}\right)=B_{2 n} /\left(1-A_{2 n}\right)$ for $n \geq 0$.

(b) By (27) $\Phi / \Psi=B_{1} /\left(1-A_{1}\right)=B_{2 n+1} /\left(1-A_{2 n+1}\right)$ for $n \geq 0$.

Now assume that $B_{n} \neq 0$ for $n \geq 0$. Then $\Phi \Psi \neq 0$. Otherwise, if $\Psi=0$ then $\Phi \neq 0$. By (26) $\Phi B_{2 n}=0$ and so $B_{2 n}=0$ for $n \geq 0$, which is a contradiction. In the other case, if $\Phi=0$ then $\Psi \neq 0$. By (27) $\Psi B_{2 n+1}=0$ and so $B_{2 n+1}=0$ for $n \geq 0$, which is a contradiction. Hence $A_{n} \neq 1$ for $n \geq 0$ and the proof follows similarly to the previous part.

(2) In view of (26) and (27) condition $A_{n}=1$ for $n \geq 0$ implies $B_{2 n} \Phi=B_{2 n+1} \Psi=0$ for $n=0,1, \ldots$

(a) Assume that $B_{2 n}=B_{2 n+1}, n=0,1, \ldots$. If $B_{2 n} \neq 0$ for some $n \geq 0$, then $B_{2 n+1} \neq 0$ for this $n$ and so $\Phi=\Psi=0$, which is a contradiction. Hence $B_{2 n}=0$ for $n \geq 0$. Thus $B_{2 n}=B_{2 n+1}=$ $0, n=0,1, \ldots$

(b) Assume that $B_{2 n} \neq B_{2 n+1}$ for $n \geq 0$.

(i) If $B_{2 n+1}=0$ for $n \geq 0$; then $B_{2 n} \neq 0$ for $n \geq 0$. Now suppose that $B_{2 n} \neq 0$ for $n \geq 0$; then from $B_{2 n} \Phi=B_{2 n+1} \Psi=0$ we get that $\Phi=$ $\Psi B_{2 n+1} / B_{2 n}=0$ for $n \geq 0$. Since $\Phi=0$, then $\Psi \neq 0$. Thus $B_{2 n+1}=0$ for $n \geq 0$.

(ii) The proof is similar to the proof of part (i).

(iii) By (26) $B_{2 n} \Phi=0$ for $n \geq 0$. If $B_{2 n} \neq 0$ for $n \geq 0$, then $\Phi=0$. Now if $B_{2 n}=0$ for $n \geq 0$, then $B_{2 n+1} \neq 0$ for $n \geq 0$. In view of (27) $\Psi B_{2 n+1}=0$ for $n \geq 0$ and so $\Psi=0$.

(3) In view of (26) and (27) condition $B_{n}=0$ for $n \geq 0$ implies $\left(1-A_{2 n}\right) \Psi=\left(1-A_{2 n+1}\right) \Phi=0$ for $n=0,1, \ldots$.

(a) Assume that $A_{2 n}=A_{2 n+1}, n=0,1, \ldots$

If $A_{2 n} \neq 1$ for some $n \geq 0$, then $A_{2 n+1} \neq 1$ for this $n$ and so $\Phi=\Psi=0$, which is a contradiction. Hence $A_{2 n}=1$ for $n \geq 0$. Thus $A_{2 n}=A_{2 n+1}=$ $1, n=0,1, \ldots$.

(b) Assume that $A_{2 n} \neq A_{2 n+1}$ for $n \geq 0$.

(i) If $A_{2 n+1}=1$ for $n \geq 0$, then $A_{2 n} \neq 1$ for $n \geq 0$. Now assume that $A_{2 n} \neq 1$ for $n \geq 0$; then from $\left(1-A_{2 n}\right) \Psi=\left(1-A_{2 n+1}\right) \Phi=0$ we get that $\Psi=\Phi\left(1-A_{2 n+1}\right) /\left(1-A_{2 n}\right)=0$ for $n \geq 0$. Since $\Psi=0$, then $\Phi \neq 0$. Thus $A_{2 n+1}=1$ for $n \geq 0$.

(ii) The proof is similar to the proof of part (i). (iii) By (26) $\left(1-A_{2 n}\right) \Psi=0$ for $n \geq 0$. If $A_{2 n} \neq 1$ for $n \geq 0$, then $\Psi=0$. Now if $A_{2 n}=1$ for $n \geq 0$, then $A_{2 n+1} \neq 1$ for $n \geq 0$. In view of (27) $\left(1-A_{2 n+1}\right) \Phi=0$ for $n \geq 0$ and so $\Phi=0$.

Corollary 14. Suppose that (1) has the linearization (2) and that $A_{n}, B_{n}$ are given by (23). Assume that (1) has a minimal period-two solution ..., $\Phi, \Psi, \ldots$, where $\Phi \neq \Psi, \Psi=$ $x_{2 n}$, and $\Phi=x_{2 n-1}$ for $n \geq 0$.

(1) Let $l \in\{1,3, \ldots\}$.

(a) $A_{n} \neq 0$ if and only if $B_{n} \neq 1, n=0,1, \ldots$

(b) If $A_{0} /\left(1-B_{0}\right)=A_{1} /\left(1-B_{1}\right)$ and either $B_{n} \neq 1$ or $A_{n} \neq 0$ for $n=0,1, \ldots$, then

(i) $B_{2 n}-A_{2 n}=1$ and $B_{2 n+1}-A_{2 n+1}=1, n=$ $0,1, \ldots$;

(ii) $\Psi=-\Phi$.

(2) Let $l \in\{2,4, \ldots\}$.

(a) $A_{n} \neq 1$ if and only if $B_{n} \neq 0, n=0,1, \ldots$

(b) If $B_{0} /\left(1-A_{0}\right)=B_{1} /\left(1-A_{1}\right)$ and either $A_{n} \neq 1$ or $B_{n} \neq 0$ for $n=0,1, \ldots$, then

(i) $A_{2 n}-B_{2 n}=1$ and $A_{2 n+1}-B_{2 n+1}=1, n=$ $0,1, \ldots$;

(ii) $\Psi=-\Phi$.

Proof. Part (1a) follows from the proof of Theorem 11 part (1). Part (1b) follows from the proof of Theorem 11 part (1) and $A_{0} /\left(1-B_{0}\right)=A_{1} /\left(1-B_{1}\right)$, which implies $\Phi / \Psi=\Psi / \Phi$. Hence $\Phi^{2}=\Psi^{2}$ and so $\Phi=-\Psi$. Thus $-1=A_{2 n} /\left(1-B_{2 n}\right)=$ $A_{2 n+1} /\left(1-B_{2 n+1}\right), n \geq 0$ which implies $B_{2 n}-A_{2 n}=1$ and $B_{2 n+1}-A_{2 n+1}=1$ for $n \geq 0$.

Part (2a) follows from the proof of Theorem 13 part (1) and the proof of part (2b) follows in a similar way as the proof of part (1b).

The next result gives a necessary and sufficient condition for the existence of a minimal period-two solution in the special case when $l=1$ in (2).

Theorem 15. Suppose that (1) has the linearization (2) with $l=1$ and that $A_{n}, B_{n}$ are as in (23). Then (1) has a minimal period-two solution if and only if one of the following holds.

(1) All the conditions of Theorem 11 part (1) are satisfied. Furthermore the period-two solution is ..., $A_{0} x_{0} /(1-$ $\left.B_{0}\right), A_{1} x_{-1} /\left(1-B_{1}\right), \ldots$

(2) $B_{n}=1$ for $n \geq 0$ and one of the following conditions is satisfied for $n \geq 0$ :

(a) $A_{2 n}=A_{2 n+1}=0$,

(b) $A_{2 n}=0$ and $A_{2 n+1} \neq 0$,

(c) $A_{2 n} \neq 0$ and $A_{2 n+1}=0$.

(3) $A_{n}=0$ for $n \geq 0$ and one of the following conditions is satisfied for $n \geq 0$ : 

(a) $B_{2 n}=B_{2 n+1}=1$,
(b) $B_{2 n}=1$ and $B_{2 n+1} \neq 1$,
(c) $B_{2 n} \neq 1$ and $B_{2 n+1}=1$.

Proof. The necessary part follows from Theorem 11. We will now prove the sufficient part.

(1) Assume that conditions (a) and (b) of Theorem 11 part (1) are satisfied. Then by (a) $x_{0}=\left(1-B_{0}\right) x_{-1} / A_{0}=$ $\left(1-B_{2 n}\right) x_{-1} / A_{2 n}$ for $n \geq 0$ and by (b) $x_{-1}=(1-$ $\left.B_{1}\right) x_{0} / A_{1}=\left(1-B_{2 n+1}\right) x_{0} / A_{2 n+1}$ for $n \geq 0$. Choose the initial conditions $x_{0}=x_{-2}=\cdots, x_{-1}=x_{-3}=\cdots$, and $x_{0} \neq x_{-1}$. Then by using these equalities we get

$$
\begin{gathered}
x_{1}=A_{0} x_{0}+B_{0} x_{-1}=\left(1-B_{0}\right) x_{-1}+B_{0} x_{-1}=x_{-1}, \\
x_{2}=A_{1} x_{-1}+B_{1} x_{0}=\left(1-B_{1}\right) x_{0}+B_{1} x_{0}=x_{0}, \\
x_{3}=A_{2} x_{0}+B_{2} x_{-1}=\left(1-B_{2}\right) x_{-1}+B_{2} x_{-1}=x_{-1}, \\
x_{4}=A_{3} x_{-1}+B_{3} x_{0}=\left(1-B_{3}\right) x_{0}+B_{3} x_{0}=x_{0} .
\end{gathered}
$$

Simple induction completes the proof.

(2) Next, suppose that $B_{n}=1$ for $n \geq 0$.

(a) Assume that $A_{2 n}=A_{2 n+1}=0$ for $n \geq 0$. By choosing the initial conditions $x_{0}=x_{-2}=$ $\cdots, x_{-1}=x_{-3}=\cdots$, and $x_{0} \neq x_{-1}$, an immediate calculation shows that $\left\{x_{-1}, x_{0}\right\}$ is a minimal period-two solution.

(b) Assume that $A_{2 n}=0$ and $A_{2 n+1} \neq 0$ for $n \geq 0$. By choosing the initial conditions $x_{0}=x_{-2}=$ $\cdots, x_{-1}=x_{-3}=\cdots=0$, and $x_{0} \neq 0$, we obtain

$$
\begin{aligned}
& x_{1}=A_{0} x_{0}+B_{0} x_{-1}=0, \\
& x_{2}=A_{1} x_{1}+B_{1} x_{0}=x_{0}, \\
& x_{3}=A_{2} x_{2}+B_{2} x_{1}=0, \\
& x_{4}=A_{3} x_{3}+B_{3} x_{2}=x_{0},
\end{aligned}
$$

and straightforward induction shows that $\left\{0, x_{0}\right\}$ is the minimal period-two solution.

(c) Assume that $A_{2 n} \neq 0$ and $A_{2 n+1}=0$ for $n \geq$ 0 . Choose the initial conditions $x_{0}=x_{-2}=$ $\cdots=0, x_{-1}=x_{-3}=\cdots$, and $x_{-1} \neq 0$. By straightforward induction we obtain that $\left\{x_{-1}, 0\right\}$ is the minimal period-two solution.

(3) Next, suppose that $A_{n}=0$ for $n \geq 0$.

(a) Assume that $B_{2 n}=B_{2 n+1}=1$ for $n \geq 0$. By choosing the initial conditions $x_{0}=x_{-2}=$ $\cdots, x_{-1}=x_{-3}=\cdots$, and $x_{0} \neq x_{-1}$, an immediate calculation shows that $\left\{x_{-1}, x_{0}\right\}$ is the minimal period-two solution. (b) Assume that $B_{2 n}=1$ and $B_{2 n+1} \neq 1$ for $n \geq 0$. By choosing the initial conditions $x_{0}=x_{-2}=\cdots=$ $0, x_{-1}=x_{-3}=\cdots$, and $x_{-1} \neq 0$, we obtain

$$
\begin{gathered}
x_{1}=A_{0} x_{0}+B_{0} x_{-1}=x_{-1}, \\
x_{2}=A_{1} x_{1}+B_{1} x_{0}=0, \\
x_{3}=A_{2} x_{2}+B_{2} x_{1}=x_{-1}, \\
x_{4}=A_{3} x_{3}+B_{3} x_{2}=0,
\end{gathered}
$$

and straightforward induction shows that $\left\{x_{-1}, 0\right\}$ is the minimal period-two solution.

(c) Assume that $B_{2 n} \neq 1$ and $B_{2 n+1}=1$ for $n \geq 0$. By choosing the initial conditions $x_{0}=x_{-2}=$ $\cdots, x_{-1}=x_{-3}=\cdots=0$, and $x_{0} \neq 0$, and using a straightforward induction we obtain that $\left\{0, x_{0}\right\}$ is the minimal period-two solution.

Example 16. The difference equation

$$
x_{n+1}=\frac{B x_{n} x_{n-1}}{d x_{n}+e x_{n-1}}, \quad n=0,1, \ldots,
$$

where $B, d, e>0$, has a minimal period-two solutions of the form $\left\{x_{-1},-x_{-1}\right\}, x_{-1} \neq 0$, if and only if $B=d-e$ and $B \neq d$.

This equation is an illustration of Theorem 15 . The linearization of (34) gives

$$
x_{n+1}=g_{1} x_{n-1}, \quad n \geq 0,
$$

where $g_{1}=B x_{n} /\left(d x_{n}+e x_{n-1}\right), n \geq 0$. Since $A_{n}=0$ for all $n \geq 0$, then from Theorem 15 part (3a) a period-two solution exists if and only if

$$
g_{1}=B_{n}=\frac{B x_{n}}{d x_{n}+e x_{n-1}}=1, \quad \forall n=0,1, \ldots
$$

Observe that if $B=d$, then $e x_{n-1}=0$ for $n \geq 0$, which is a contradiction.

Equation (36) gives the first order equation

$$
x_{n}=\frac{e}{B-d} x_{n-1}, \quad n=0,1, \ldots
$$

which has a period-two solution if and only if $B=d-e$ and $x_{0}=-x_{-1}$ as Theorem 15 part (1) is satisfied.

Therefore, (34) has a period-two solution of the form $\left\{x_{-1},-x_{-1}\right\}, x_{-1} \neq 0$ if and only if $B=d-e, B \neq d$.

This example can be extended to a more general equation of the form

$$
\begin{array}{r}
x_{n+1}=\frac{B x_{n} x_{n-1} x_{n-2}}{a_{0} x_{n-1} x_{n-2}+a_{1} x_{n} x_{n-2}+a_{2} x_{n} x_{n-1}}, \\
n=0,1, \ldots,
\end{array}
$$

where $B, a_{i}>0, i=0,1,2$. Similar reasoning gives the necessary and sufficient conditions for the existence of a period-two solution to be

$$
B=a_{1}-\left(a_{0}+a_{2}\right), \quad B \neq a_{1} .
$$

In this case there is an infinite number of period-two solutions of the form $\left\{x_{-1},-x_{-1}\right\}, x_{-1} \neq 0$. 
Corollary 17. Suppose that (1) has the linearization (2) with $l=1$ and that $A_{n}, B_{n}$ are as in (23). Assume that $A_{0} /\left(1-B_{0}\right)=$ $A_{1} /\left(1-B_{1}\right)$ and either $B_{n} \neq 1$ or $A_{n} \neq 0$ for $n \geq 0$. Then (1) has a minimal period-two solution if and only if $B_{2 n+1}-A_{2 n+1}=$ 1 and $B_{2 n}-A_{2 n}=1$ for $n \geq 0$.

Proof. The necessary part follows from Corollary 14 part (1b).

For the proof of sufficient part choose the initial conditions $x_{0}=x_{-2}=\cdots, x_{-1}=x_{-3}=\cdots$, and $x_{0}=-x_{-1}$. Setting $n=0,1, \ldots$ we obtain

$$
\begin{aligned}
& x_{1}=A_{0} x_{0}+B_{0} x_{-1}=-A_{0} x_{-1}+B_{0} x_{-1}=x_{-1}, \\
& x_{2}=A_{1} x_{1}+B_{1} x_{0}=A_{1} x_{-1}-B_{1} x_{-1}=-x_{-1}, \\
& x_{3}=A_{2} x_{2}+B_{2} x_{1}=-A_{2} x_{-1}+B_{2} x_{-1}=x_{-1}, \\
& x_{4}=A_{3} x_{3}+B_{3} x_{2}=A_{3} x_{-1}-B_{3} x_{-1}=-x_{-1} .
\end{aligned}
$$

Simple induction completes the proof.

So far we have considered the cases when $l \in\{1,3, \ldots\}$ and $B_{n}$ is either equal or not equal to one for all $n \geq 0$. But what happens when $l \in\{1,3, \ldots\}$ and $B_{n}=1$ for some $n$ 's and $B_{n} \neq 1$ for other $n$ 's or when $A_{n}=0$ for some $n$ 's and $A_{n} \neq 0$ for other $n$ 's? We will now investigate these cases along with the cases when $l \in\{2,4, \ldots\}$ and $B_{n}=0$ for some $n$ 's and $B_{n} \neq 0$ for other $n$ 's or when $A_{n}=1$ for some $n$ 's and $A_{n} \neq 1$ for other $n$ 's.

Theorem 18. Let $l \in\{1,3, \ldots\}$. Suppose that (1) has the linearization (2) and that $A_{n}, B_{n}$ are as in (23). Assume that (1) has a minimal period-two solution ..., $\Phi, \Psi, \ldots$, where $\Phi \neq \Psi, \Psi=x_{2 n}$, and $\Phi=x_{2 n-1}$ for $n \geq 0$.

(1) Let $\Phi \Psi \neq 0$. Then

(a) for some $N \in\{0,1, \ldots\} \quad B_{N}=1$ if and only if $A_{N}=0$;

(b) if some $N$ are even and $B_{N} \neq 1$, then $A_{N}=(\Phi / \Psi)$ $\left(1-B_{N}\right)$;

(c) if some $N$ are odd and $B_{N} \neq 1$, then $A_{N}=(\Psi / \Phi)$ $\left(1-B_{N}\right)$.

(2) If $\Psi=0$, then for all $n \geq 0 B_{2 n}=1$ and $A_{2 n+1}=0$.

(3) If $\Phi=0$, then for all $n \geq 0 B_{2 n+1}=1$ and $A_{2 n}=0$.

Proof. The proof is as follows.

(1) The result follows from Lemma 9 part (a).

(2) Since $\Psi=0$, then $\Phi \neq 0$ and the result follows from Lemma 9 part (a).

(3) Since $\Phi=0$, then $\Psi \neq 0$ and the result follows from Lemma 9 part (a).

Remark 19. Note that in part (2) of Theorem $18 B_{2 n+1} \in$ $\mathbb{R}$ and $A_{2 n} \in \mathbb{R}$ for all $n \geq 0$. Similarly in part (3) of Theorem $18 B_{2 n} \in \mathbb{R}$ and $A_{2 n+1} \in \mathbb{R}$ for all $n \geq 0$.
Theorem 20. Let $l \in\{2,4, \ldots\}$. Suppose that (1) has the linearization (2) and that $A_{n}, B_{n}$ are as in (23). Assume that (1) has a minimal period-two solution ..., $\Phi, \Psi, \ldots$, where $\Phi \neq \Psi, \Psi=x_{2 n}$, and $\Phi=x_{2 n-1}$ for $n \geq 0$.

(1) Let $\Phi \Psi \neq 0$. Then

(a) for some $N \in\{0,1, \ldots\} A_{N}=1$ if and only if $B_{N}=0$;

(b) if some $N$ are even and $A_{N} \neq 1$, then $B_{N}=$ $(\Psi / \Phi)\left(1-A_{N}\right)$;

(c) if some $N$ are odd and $A_{N} \neq 1$, then $B_{N}=$ $(\Phi / \Psi)\left(1-A_{N}\right)$

(2) If $\Psi=0$, then for all $n \geq 0 B_{2 n}=0$ and $A_{2 n+1}=1$.

(3) If $\Phi=0$, then for all $n \geq 0 B_{2 n+1}=0$ and $A_{2 n}=1$.

Proof. The results follow from Lemma 9 part (b).

Theorem 21. Suppose that (1) has the linearization (2) with $l=$ 1 and that $A_{n}, B_{n}$ are as in (23). Then (1) has a minimal periodtwo solution ..., $\Phi, \Psi, \ldots$, where $\Phi \neq \Psi, \Psi=x_{2 n}$, and $\Phi=$ $x_{2 n-1}$ for $n \geq 0$ if and only if one of the following holds:

(1) $\Phi \Psi \neq 0$ and for each $n \geq 0$ either

(a) $B_{n}=1$ and $A_{n}=0$;

(b) $n$ is even, $B_{n} \neq 1$, and $A_{n}=(\Phi / \Psi)\left(1-B_{n}\right)$;

(c) $n$ is odd, $B_{n} \neq 1$, and $A_{n}=(\Psi / \Phi)\left(1-B_{n}\right)$.

(2) If $\Psi=0$, then for all $n \geq 0 B_{2 n}=1$ and $A_{2 n+1}=0$.

(3) If $\Phi=0$, then for all $n \geq 0 B_{2 n+1}=1$ and $A_{2 n}=0$.

Proof. The necessary part follows from Theorem 18. The sufficient part follows by direct calculation of the period-two solution which satisfies specific initial conditions in a similar way as in the proof of Theorem 15.

An illustration of Theorem 20 is the following example.

Example 22. The difference equation

$$
x_{n+1}=\frac{\sin \left(x_{n-1}\right)}{n+1} x_{n}+x_{n-1} e^{x_{n}}, \quad n=0,1, \ldots,
$$

has an infinite number of period-two solutions of the form $\left\{0, x_{0}\right\}, x_{0} \neq 0$, which can be seen by immediate checking. In view of Theorem 20 part (3) $A_{2 n}=\sin \left(x_{2 n-1}\right) /(2 n+1)$ and $B_{2 n+1}=e^{x_{2 n+1}}=1$ for $n=0,1, \ldots$ when $\Phi=0$.

\section{Conflict of Interests}

The authors declare that there is no conflict of interests regarding the publication of this paper.

\section{References}

[1] E. J. Janowski and M. R. S. Kulenović, "Attractivity and global stability for linearizable difference equations," Computers \& Mathematics with Applications, vol. 57, no. 9, pp. 1592-1607, 2009. 
[2] E. J. Janowski, M. R. S. Kulenović, and E. Silić, "Periodic solutions of linearizable difference equations," International Journal of Difference Equations, vol. 6, no. 2, pp. 113-125, 2011.

[3] S. Elaydi, An Introduction to Difference Equations, Undergraduate Texts in Mathematics, Springer, New York, NY, USA, 3rd edition, 2005.

[4] M. R. S. Kulenović and O. Merino, Discrete Dynamical Systems and Difference Equations with Mathematica, Chapman \& Hall/CRC, London, UK, 2002.

[5] H. Sedaghat, Nonlinear Difference Equations, Theory with Applications to Social Science Models, vol. 15 of Mathematical Modelling: Theory and Applications, Kluwer Academic, Dordrecht, Germany, 2003.

[6] A. M. Amleh, E. Camouzis, and G. Ladas, "On the dynamics of a rational difference equation, part I," International Journal of Difference Equations, vol. 3, no. 1, pp. 1-35, 2008.

[7] J. K. Hale and H. Koçak, Dynamics and Bifurcations, vol. 3 of Texts in Applied Mathematics, Springer, New York, NY, USA, 1991.

[8] V. L. Kocić and G. Ladas, Global Behavior of Nonlinear Difference Equations of Higher Order with Applications, Kluwer Academic, Dordrecht, Germany, 1993.

[9] M. R. S. Kulenović and G. Ladas, Dynamics of Second Order Rational Difference Equations, with Open Problems and Conjectures, Chapman \& Hall/CRC, Boca Raton, Fla, USA, 2001.

[10] M. R. S. Kulenović and O. Merino, "Global bifurcation for discrete competitive systems in the plane," Discrete and Continuous Dynamical Systems B, vol. 12, no. 1, pp. 133-149, 2009.

[11] A. M. Amleh, E. A. Grove, G. Ladas, and D. A. Georgiou, "On the recursive sequence $x_{n+1}=\alpha+x_{n-1} / x_{n}$ " Journal of Mathematical Analysis and Applications, vol. 233, no. 2, pp. 790798, 1999.

[12] C. H. Gibbons, M. R. S. Kulenović, G. Ladas, and H. D. Voulov, "On the trichotomy character of $x_{n+1}=\left(\alpha+\beta x_{n}+\gamma x_{n-1}\right) /(A+$ $\left.x_{n}\right)$," Journal of Difference Equations and Applications, vol. 8, no. 1, pp. 75-92, 2002.

[13] T. Nesemann, "Invariants and Liapunov functions for nonautonomous systems. Advances in difference equations, III," Computers \& Mathematics with Applications, vol. 42, no. 3-5, pp. 385-392, 2001.

[14] C. C. McCluskey and J. S. Muldowney, "Bendixson-Dulac criteria for difference equations," Journal of Dynamics and Differential Equations, vol. 10, no. 4, pp. 567-575, 1998.

[15] C. C. McCluskey and J. S. Muldowney, "Stability implications of Bendixson conditions for difference equations," in Proceedings of the 6th International Conference on Difference Equations, pp. 181-187, CRC, Boca Raton, Fla, USA, 2004. 


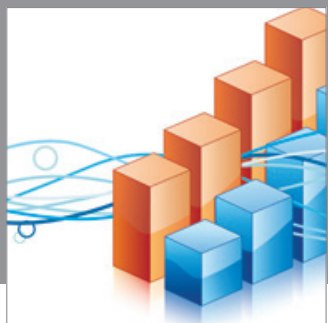

Advances in

Operations Research

mansans

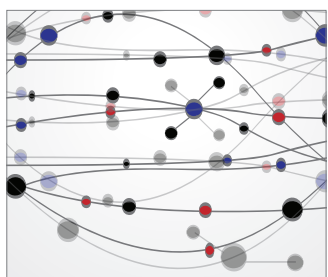

The Scientific World Journal
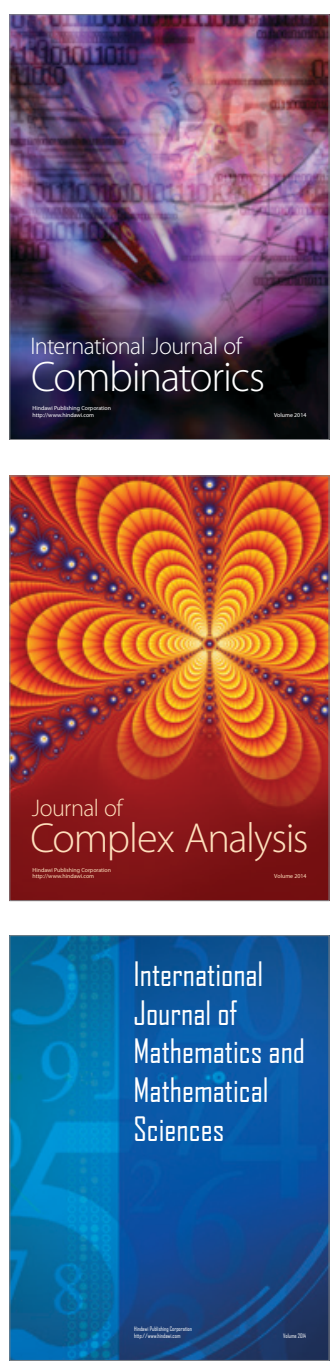
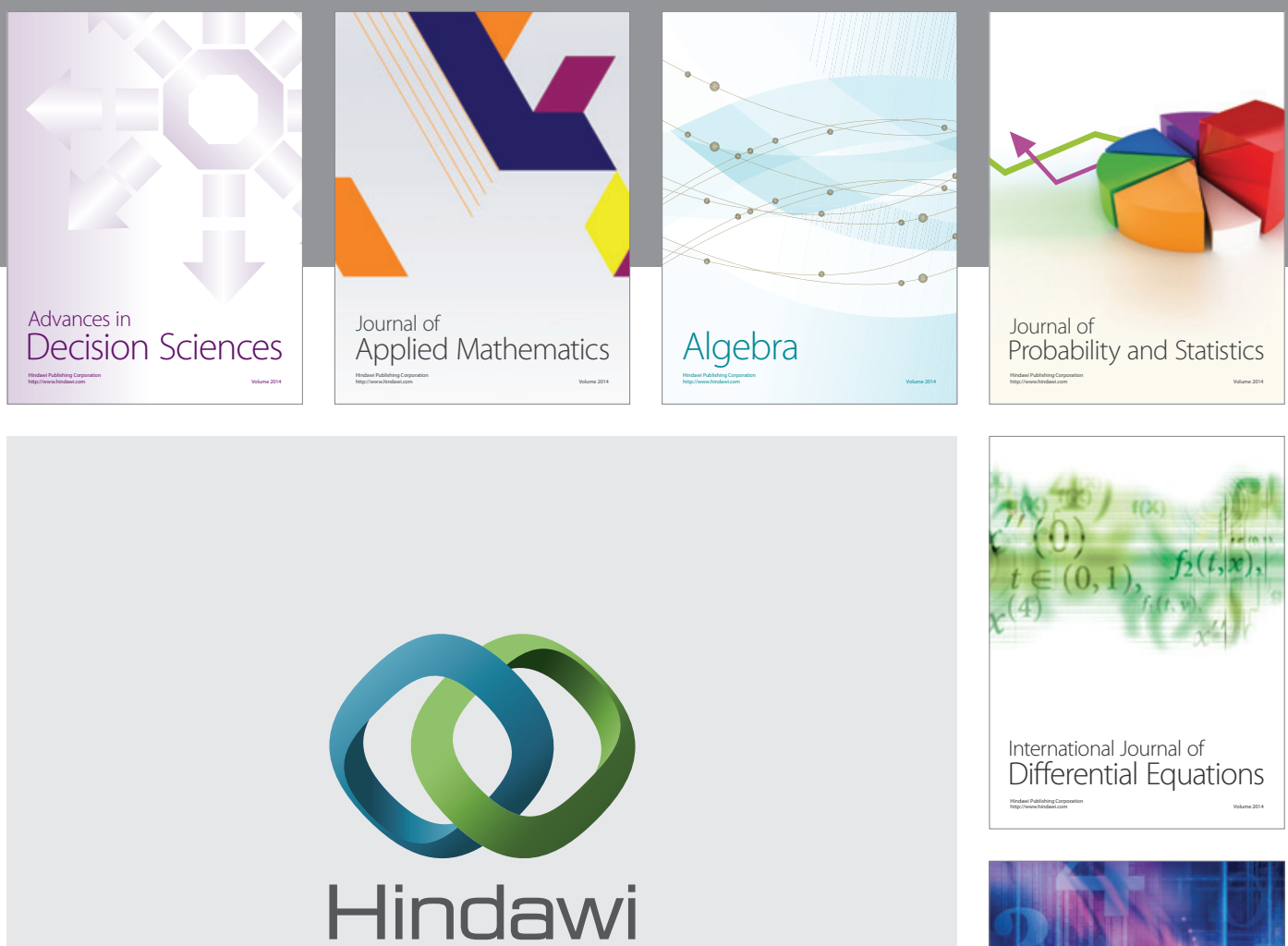

Submit your manuscripts at http://www.hindawi.com
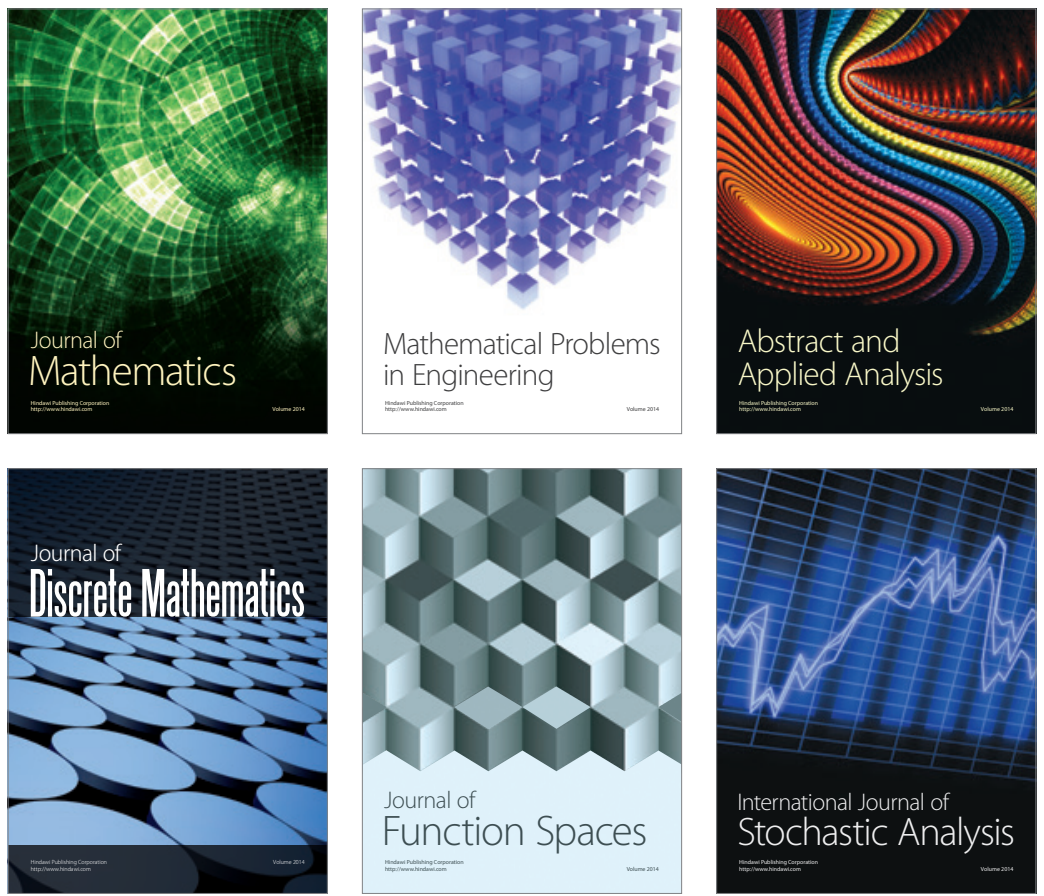

Journal of

Function Spaces

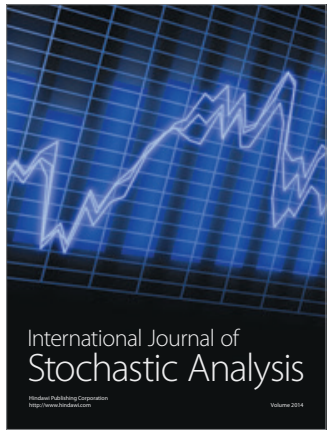

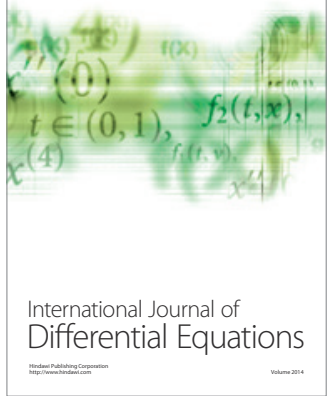
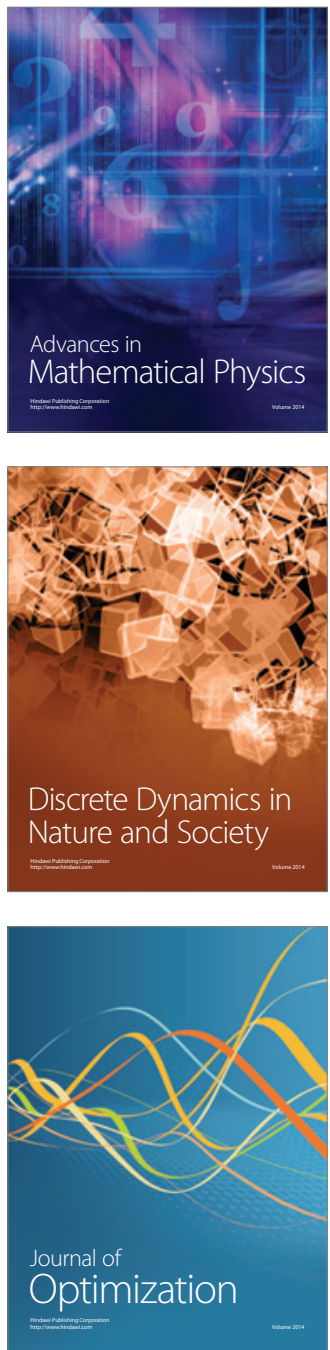